CALT-TH-2020-056

\title{
STrEAMlining EFT Matching
}

\author{
Timothy Cohen, ${ }^{1}$ Xiaochuan Lu, ${ }^{1}$ and Zhengkang Zhang ${ }^{2}$ \\ ${ }^{1}$ Institute for Fundamental Science, University of Oregon, Eugene, OR 97403 \\ ${ }^{2}$ Walter Burke Institute for Theoretical Physics, California Institute of Technology, Pasadena, CA 91125 \\ E-mail: tcohen@uoregon.edu, xlu@uoregon.edu, zkzhang@caltech.edu
}

\begin{abstract}
This paper presents STrEAM (SuperTrace Evaluation Automated for Matching), a Mathematica package that calculates all functional supertraces which arise when matching a generic UV model onto a relativistic Effective Field Theory (EFT) at one loop and to arbitrary order in the heavy mass expansion. STrEAM implements the covariant derivative expansion to automate the most tedious step of the streamlined functional matching prescription presented in Ref. [1]. The code and an example notebook are available at this link. (
\end{abstract}




\section{Contents}

1 Introduction 2

2 Scope of STrEAM 3

3 Algorithm $\quad 6$

3.1 CDE Review 6

3.2 CDE Within STrEAM 9

$\begin{array}{ll}3.3 & \text { Implementation Summary } \\ 3.41\end{array}$

3.4 A Simple Example 12

4 STrEAM Manual $\quad 14$

$\begin{array}{lr}\text { References } & 19\end{array}$

\section{Introduction}

Effective Field Theories (EFTs) provide a useful and convenient framework for describing the dynamics of low-energy degrees of freedom in a model-independent way, see e.g. Refs. [2-10] for reviews. When the more fundamental UV theory is known (and calculable), one can integrate out the heavy physics and derive an EFT description valid at low energies. This so-called "matching" calculation links the Wilson coefficients in the EFT with the microscopic parameters of the UV theory.

EFT matching can be efficiently performed with functional methods [11-33]. In Ref. [1], we provided a STrEAMlined functional prescription for relativistic EFT matching up to one-loop level. Functional supertraces play an essential role; enumerating and evaluating them are the key steps in this prescription. Enumeration of the functional supertraces can be performed graphically as discussed in Ref. [1]. Evaluation can be efficiently achieved using the Covariant Derivative Expansion (CDE) technique [11-13]. This is a straightforward procedure that becomes tedious when the supertrace is complicated and/or a high operator dimension is desired. In this paper, we address this problem by introducing a Mathematica package, STrEAM (SuperTrace Evaluation Automated for Matching), which automates this procedure ( $)$ [34].

Many automated tools for EFT calculations, especially in the context of the Standard Model EFT, have been developed in recent years [35-48], see Ref. [49] for a summary. Among them, MatchingTools [37] addresses EFT matching at tree level, 
while MatchMaker [48] (not yet released) automates Feynman diagram matching up to one-loop level. Codes are also available for partially computing one-loop EFT Lagrangians in the framework of the Universal One-Loop Effective Action [29, 31, 40]. To our knowledge, STrEAM is the first publicly available package that automates functional supertrace evaluations for general one-loop EFT matching calculations to arbitrary order in the heavy mass expansion. ${ }^{1}$

The rest of this paper is organized as follows. In Sec. 2, we explain the scope of STrEAM, i.e., the specific form of functional supertraces that it evaluates; these include the two types of supertraces that appear in general one-loop functional matching calculations. In Sec. 3, we review the CDE technique and explain how to apply it to the form of supertraces targeted by STrEAM. We summarize the implementation of CDE in STrEAM and provide a simple example to demonstrate this procedure. Sec. 4 provides a user manual for STrEAM.

\section{Scope of STrEAM}

In this section, we discuss the motivation and specify the scope of STrEAM. We first review the types of functional supertraces that could arise from one-loop relativistic EFT matching calculations. We then describe the precise form of functional supertraces that STrEAM evaluates and explain why it covers all the possible supertraces that can appear when performing one-loop functional matching.

Consider a UV theory $\mathcal{L}_{\mathrm{UV}}[\Phi, \phi]$ with a mass hierarchy among its fields,

$$
m_{\Phi} \gg m_{\phi}
$$

We would like to integrate out the heavy fields $\Phi$ to derive an EFT for the light fields $\mathcal{L}_{\mathrm{EFT}}[\phi]$. As elaborated in Ref. [1], one-loop matching with functional methods receives contributions from two types of supertraces:

$$
\int \mathrm{d}^{d} x \mathcal{L}_{\mathrm{EFT}}^{(1-\text { loop })}[\phi]=\left.\frac{i}{2} \mathrm{~S} \operatorname{Tr} \log \boldsymbol{K}\right|_{\text {hard }}-\left.\frac{i}{2} \sum_{n=1}^{\infty} \frac{1}{n} \mathrm{~S} \operatorname{Tr}\left[\left(\boldsymbol{K}^{-1} \boldsymbol{X}\right)^{n}\right]\right|_{\text {hard }}
$$

We call these "log-type" and "power-type" supertraces respectively. Here "| to extract the hard region contributions $[51,52]$ in the loop integrals arising from these supertraces. $\boldsymbol{K}$ and $\boldsymbol{X}$ are matrices acting on the space of the field multiplet

$$
\varphi \equiv\left(\begin{array}{l}
\Phi \\
\phi
\end{array}\right)
$$

\footnotetext{
${ }^{1}$ As this project was reaching completion, we became aware of the program "SuperTracer," to be released simultaneously [50].
} 
We refer the reader to Ref. [1] for detailed definitions, as well as a discussion on how to derive $\boldsymbol{K}$ and $\boldsymbol{X}$ from the UV Lagrangian.

Let us first examine the log-type supertraces. In relativistic theories, the matrix $\boldsymbol{K}$ has a block-diagonal form with entries

$$
K_{i}= \begin{cases}P^{2}-m_{i}^{2} & \left(\varphi_{i} \text { is spin- } 0\right) \\ \not P-m_{i} & \left(\varphi_{i} \text { is spin- } \frac{1}{2}\right) \\ -\eta^{\mu \nu}\left(P^{2}-m_{i}^{2}\right) & \left(\varphi_{i} \text { is spin- }\right)\end{cases}
$$

where $P_{\mu} \equiv i D_{\mu}$ is the Hermitian covariant derivative. Because of the hard region requirement, only heavy field components $\varphi_{i} \in\{\Phi\}$ yield nonzero log-type supertraces. Up to overall constants, they are universally given by

$$
i \mathrm{~S} \operatorname{Tr} \log K_{\Phi}= \begin{cases}i \mathrm{~S} \operatorname{Tr} \log \left(P^{2}-m_{\Phi}^{2}\right) & (\Phi \text { is spin-0 or spin- } 1) \\ i \mathrm{~S} \operatorname{Tr} \log \left(\not P-m_{\Phi}\right) & \left(\Phi \text { is spin- } \frac{1}{2}\right)\end{cases}
$$

The power-type supertraces, on the other hand, are not universal, since they require specifying the interaction matrix $\boldsymbol{X}$. This matrix can be written as an expansion:

$$
\boldsymbol{X}\left(\phi, P_{\mu}\right)=\boldsymbol{U}[\phi]+\left(P_{\mu} \boldsymbol{Z}^{\mu}[\phi]+\overline{\boldsymbol{Z}}^{\mu}[\phi] P_{\mu}\right)+\cdots,
$$

where $P_{\mu}$ is an "open" covariant derivative. The matrix entries $U_{i j}[\phi], Z_{i j}^{\mu}[\phi], \bar{Z}_{i j}^{\mu}[\phi]$, etc. may also contain covariant derivatives, but they are closed: when viewed as operators acting on the functional space, where $\{|x\rangle\}$ and $\{|q\rangle\}$ each forms a basis and $\hat{x}$ and $\hat{q}$ are the basic position and momentum operators, $U_{i j}[\phi], Z_{i j}^{\mu}[\phi], \bar{Z}_{i j}^{\mu}[\phi]$, etc., are built out of $\hat{x}$ only. In contrast, an open covariant derivative $P_{\mu}$ is built out of both $\hat{x}$ and $\hat{q}$ :

$$
P_{\mu}(\hat{x}, \hat{q})=\hat{q}_{\mu}+g_{a} G_{\mu}^{a}(\hat{x}) T^{a},
$$

with $\hat{q}_{\mu}=i \partial_{\mu}$ in position space. In this paper, we will frequently use the notation $U_{k}(\hat{x})$ (or simply $U(\hat{x})$ ) to denote a general functional operator that is built out of $\hat{x}$ only. It can represent any of $U_{i j}[\phi], Z_{i j}^{\mu}[\phi], \bar{Z}_{i j}^{\mu}[\phi]$, etc. in Eq. (2.6):

$$
U_{k} \in\left\{U_{i j}[\phi], Z_{i j}^{\mu}[\phi], \bar{Z}_{i j}^{\mu}[\phi], \ldots\right\}
$$

With this notation, an arbitrary term in the expansion of the matrix entry $X_{i j}$ can be expressed in the form

$$
\left(P_{\mu_{1}} \cdots P_{\mu_{n}}\right) U_{k}\left(P_{\nu_{1}} \cdots P_{\nu_{m}}\right)
$$


and therefore a general term in the power-type supertraces in Eq. (2.2),

$$
-i \mathrm{~S} \operatorname{Tr}\left[\frac{1}{K_{i_{1}}} X_{i_{1} i_{2}} \frac{1}{K_{i_{2}}} X_{i_{2} i_{3}} \cdots \frac{1}{K_{i_{n}}} X_{i_{n} i_{1}}\right]
$$

is evaluated over a product sequence of segments of the form

$$
\frac{1}{K_{i}}\left(P_{\mu_{1}} \cdots P_{\mu_{n}}\right) U_{k}\left(P_{\nu_{1}} \cdots P_{\nu_{m}}\right)
$$

In what follows, we use $\Delta_{i}$ and $\Lambda_{i}$ to denote the bosonic and fermionic versions of $K_{i}^{-1}$, respectively:

$$
\Delta_{i} \equiv \frac{1}{P^{2}-m_{i}^{2}}, \quad \Lambda_{i} \equiv \frac{1}{\not P-m_{i}}
$$

STrEAM automates the evaluation of functional supertraces of the form

$$
-\left.i \mathrm{~S} \operatorname{Tr}\left[f\left(P_{\mu},\left\{U_{k}\right\}\right)\right]\right|_{\text {hard }}
$$

where $f$ is a product sequence of $P_{\mu}, U_{k}, \Delta_{i}$ and $\Lambda_{i}$, consisting of an arbitrary number of "propagator blocks":

$$
f=\left[\cdots\left(P_{\mu_{1}} \ldots P_{\mu_{n}}\right)\left(\Delta_{i} \text { or } \Lambda_{i}\right)\left(P_{\nu_{1}} \ldots P_{\nu_{m}}\right) U_{k} \cdots\right]
$$

As indicated by the name, each "propagator block" has a propagator as its central object, which can be either $\Delta_{i}$ or $\Lambda_{i}$. There can be an arbitrary number of additional open covariant derivatives $P_{\mu}$ surrounding the propagator. A propagator block terminates with a $U$ factor, after which the next block starts. From the discussion above, it is clear that this form of $f$ covers any possible power-type supertraces. ${ }^{2}$ We further allow the last block in $f$ to have a trivial $U$ factor, i.e., $U=1$. In this way, the log-type supertraces in Eq. (2.5) can also be covered upon taking a mass derivative

$$
\begin{gathered}
\frac{\partial}{\partial m_{\Phi}^{2}}\left[i \mathrm{~S} \operatorname{Tr} \log \left(P^{2}-m_{\Phi}^{2}\right)\right]=-i \mathrm{~S} \operatorname{Tr}\left[\frac{1}{P^{2}-m_{\Phi}^{2}}\right]=-\left.i \mathrm{~S} \operatorname{Tr}\left[\Delta_{\Phi}\right]\right|_{\text {hard }}, \\
\frac{\partial}{\partial m_{\Phi}}\left[i \mathrm{~S} \operatorname{Tr} \log \left(\not P-m_{\Phi}\right)\right]=-i \mathrm{~S} \operatorname{Tr}\left[\frac{1}{\not P-m_{\Phi}}\right]=-\left.i \mathrm{~S} \operatorname{Tr}\left[\Lambda_{\Phi}\right]\right|_{\text {hard }} .
\end{gathered}
$$

Next, we describe the CDE method for evaluating these supertraces.

\footnotetext{
${ }^{2}$ If the last $\frac{1}{K_{i_{n}}} X_{i_{n} i_{1}}$ segment in Eq. (2.10) has $P_{\mu}$ factors after the $U_{k}$ factor (see Eq. (2.11)), one can cyclicly permute them to the beginning of the expression, namely before $\frac{1}{K_{i_{1}}}$, and then use STrEAM to evaluate it.
} 


\section{Algorithm}

STrEAM implements CDE as its central algorithm to evaluate the supertraces. This technique was originally developed in Refs. [11-13], and applied to modern EFT matching calculations in Refs. [14, 15]; a variant was also developed later in Refs. [20, 22, 23]. A comprehensive review of both versions of the CDE can be found in App. B of Ref. [30], where they were termed "original CDE" and "simplified CDE" respectively. The algorithm implemented in STrEAM is the original CDE; see App. B.2.3 of Ref. [30]. In this section, we briefly review it with a focus on how it is implemented in STrEAM.

\subsection{CDE Review}

The simplified CDE and original CDE are techniques that one can use to evaluate a functional supertrace

$$
-i \operatorname{STr}\left[f\left(P_{\mu},\left\{U_{k}\right\}\right)\right]=\int \mathrm{d}^{d} x \sum_{i} c_{i} \mathcal{O}_{i}(x) .
$$

Here $f$ is an operator in the functional space, built out of the covariant derivative $P_{\mu}(\hat{x}, \hat{q})$ (see Eq. (2.7)) and a set of functions $\left\{U_{k}(\hat{x})\right\}$ (as well as constants that we will suppress). In this paper, we are eventually interested in the form of $f$ evaluated by STrEAM, i.e., Eq. (2.14). However, the method that we review in this subsection applies to a broader class of $f$, for which we only require a well-defined power expansion in its arguments $P_{\mu}$ and $\left\{U_{k}\right\}$ about $P_{\mu}=\left\{U_{k}\right\}=0$. For example, the log-type supertraces in Eq. (2.5) give $f=\log \left(P^{2}-m_{\Phi}^{2}\right)$ or $f=\log \left(\not P-m_{\Phi}\right)$; they also satisfy this condition. Nevertheless, it is useful to think of the form in Eq. (2.14) as a benchmark example of $f$. The general evaluation yields a set of local operators $\mathcal{O}_{i}$ integrated over spacetime. In the context of EFT matching, we call $\mathcal{O}_{i}$ "effective operators" and $c_{i}$ "Wilson coefficients," although we emphasize that functional supertrace evaluation can also be used to derive one-particle-irreducible effective actions more generally.

First, we address the "super" part of the functional supertrace. This part gives an overall sign \pm depending on whether the diagonal entry comes from a bosonic or fermionic field $\varphi_{i}$ :

$$
-i \operatorname{STr}\left[f\left(P_{\mu},\left\{U_{k}\right\}\right)\right]= \pm\left\{-i \operatorname{Tr}\left[f\left(P_{\mu},\left\{U_{k}\right\}\right)\right]\right\} .
$$

Given the form of $f$ in Eq. (2.14), one can determine this overall sign from the first propagator in the product sequence. If it is $\Delta_{i}\left(\Lambda_{i}\right)$, then the entry comes from a bosonic (fermionic) $\varphi_{i}$, and we should take the $+(-)$ sign in Eq. (3.2). The only exception is that a Faddeev-Popov ghost gives a propagator $\Delta_{i}$, but it is a fermionic 
field. In this case, one should take the minus sign option in Eq. (3.2).

Next, we deal with the more difficult "functional" part of the functional supertrace. We start out with its definition, and then make an insertion of unity in the functional space $\mathbf{1}=\int \mathrm{d}^{d} x|x\rangle\langle x|$ :

$$
\begin{aligned}
-i \operatorname{Tr}\left[f\left(P_{\mu},\left\{U_{k}\right\}\right)\right] & =-i \int \frac{\mathrm{d}^{d} q}{(2 \pi)^{d}}\left\langle q\left|\operatorname{tr}\left[f\left(P_{\mu},\left\{U_{k}\right\}\right)\right]\right| q\right\rangle \\
& =-i \int \mathrm{d}^{d} x \int \frac{\mathrm{d}^{d} q}{(2 \pi)^{d}}\langle q \mid x\rangle\left\langle x\left|\operatorname{tr}\left[f\left(P_{\mu},\left\{U_{k}\right\}\right)\right]\right| q\right\rangle \\
& =-i \int \mathrm{d}^{d} x \int \frac{\mathrm{d}^{d} q}{(2 \pi)^{d}} e^{i q \cdot x} \operatorname{tr}\left[f\left(P_{\mu},\left\{U_{k}\right\}\right)\right] e^{-i q \cdot x}
\end{aligned}
$$

In the last line, we have used

$$
\langle q \mid x\rangle=(\langle x \mid q\rangle)^{*}=e^{i q \cdot x}
$$

Given that $f\left(P_{\mu},\left\{U_{k}\right\}\right)$ has a well defined power expansion in terms of its arguments $P_{\mu}$ and $\left\{U_{k}\right\}$, we can write

$$
e^{i q \cdot x} f\left(P_{\mu},\left\{U_{k}\right\}\right) e^{-i q \cdot x}=f\left(e^{i q \cdot x} P_{\mu} e^{-i q \cdot x},\left\{e^{i q \cdot x} U_{k} e^{-i q \cdot x}\right\}\right),
$$

because this is true for any term in the power expansion (upon making further insertions):

$$
e^{i q \cdot x}\left(A B C^{-1} \cdots\right) e^{-i q \cdot x}=\left[\left(e^{i q \cdot x} A e^{-i q \cdot x}\right)\left(e^{i q \cdot x} B e^{-i q \cdot x}\right)\left(e^{i q \cdot x} C e^{-i q \cdot x}\right)^{-1} \cdots\right] .
$$

Next, we use

$$
\begin{aligned}
& e^{i q \cdot x} P_{\mu} e^{-i q \cdot x}=P_{\mu}+q_{\mu}, \\
& e^{i q \cdot x} U_{k} e^{-i q \cdot x}=U_{k},
\end{aligned}
$$

to find

$$
-i \operatorname{Tr}\left[f\left(P_{\mu},\left\{U_{k}\right\}\right)\right]=-i \int \mathrm{d}^{d} x \int \frac{\mathrm{d}^{d} q}{(2 \pi)^{d}} \operatorname{tr}\left[f\left(P_{\mu}-q_{\mu},\left\{U_{k}\right\}\right)\right] .
$$

Here we have also flipped the sign of the integration variable $q_{\mu}$ for future convenience.

At this point, one can already make a Taylor expansion of $f\left(P_{\mu}-q_{\mu},\left\{U_{k}\right\}\right)$ in terms of the covariant derivative $P_{\mu}$ to obtain the effective operators (truncated according to the desired operator dimension). When performing such an expansion, each effective operator will be multiplied by an expression of the loop momentum $q_{\mu}$, which we will call the " $q$-section" factor. Carrying out the loop integral $-i \int \frac{\mathrm{d}^{d} q}{(2 \pi)^{d}}$ 
over the $q$-sections gives the Wilson coefficients of the effective operators. This is the "simplified CDE" method (see App. B.2.2 of Ref. [30] for more details).

In the simplified CDE, Taylor expanding $f\left(P_{\mu}-q_{\mu},\left\{U_{k}\right\}\right)$ generates effective operators in which the covariant derivatives could be either open or closed. In the end, only effective operators with closed covariant derivatives will be nonzero after performing the loop integral $-i \int \frac{\mathrm{d}^{d} q}{(2 \pi)^{d}}$; these effective operators are gauge singlets. Effective operators containing open covariant derivatives are not gauge singlets, as these open covariant derivatives eventually act on the unity function $\mathbb{1}$ which yields explicit gauge fields. They always drop out upon evaluating the loop integral $-i \int \frac{\mathrm{d}^{d} q}{(2 \pi)^{d}}$ with dimensional regularization, because the integrands, i.e., their accompanying $q-$ sections, are total derivatives in $q_{\mu}$.

There is in fact a way to systematically avoid effective operators containing open covariant derivatives at the point of making the expansion, i.e., before performing the loop integral $-i \int \frac{\mathrm{d}^{d} q}{(2 \pi)^{d}}$. This is achieved by making two further insertions in Eq. (3.8), before and after the factor $f\left(P_{\mu}-q_{\mu},\left\{U_{k}\right\}\right)$ :

$$
-i \operatorname{Tr}\left[f\left(P_{\mu},\left\{U_{k}\right\}\right)\right]=-i \int \mathrm{d}^{d} x \int \frac{\mathrm{d}^{d} q}{(2 \pi)^{d}} e^{P \cdot \frac{\partial}{\partial q}} \operatorname{tr}\left[f\left(P_{\mu}-q_{\mu},\left\{U_{k}\right\}\right)\right] e^{-P \cdot \frac{\partial}{\partial q}} .
$$

This is the "original CDE" method (see App. B.2.3 of Ref. [30] for more details). Here the second insertion is allowed because the $q_{\mu}$ derivatives in $e^{-P \cdot \frac{\partial}{\partial q}}$ act on the unity function $\mathbb{1}$ to yield zero, and hence only the first term in its Taylor expansion contributes:

$$
e^{-P \cdot \frac{\partial}{\partial q}} \mathbb{1}=\mathbb{1}
$$

The first insertion in Eq. (3.9) is allowed because all but its first term would yield total derivatives in $q_{\mu}$ and hence drop out upon evaluating the loop integral $-i \int \frac{\mathrm{d}^{d} q}{(2 \pi)^{d}}$ with dimensional regularization.

Using the same argument as in Eqs. (3.5) and (3.6), these insertions can be passed to the arguments of $f\left(P_{\mu}-q_{\mu},\left\{U_{k}\right\}\right)$, changing them into

$$
\begin{aligned}
P_{\mu}^{\mathrm{CDE}} & \equiv e^{P \cdot \frac{\partial}{\partial q}}\left(P_{\mu}-q_{\mu}\right) e^{-P \cdot \frac{\partial}{\partial q}}=-q_{\mu}+G_{\mu \nu}^{\mathrm{CDE}} \partial^{\nu} \\
U_{k}^{\mathrm{CDE}} & \equiv e^{P \cdot \frac{\partial}{\partial q}} U_{k} e^{-P \cdot \frac{\partial}{\partial q}}=\sum_{n=0}^{\infty} \frac{1}{n !}\left(P_{\alpha_{1}} \cdots P_{\alpha_{n}} U_{k}\right) \partial^{\alpha_{1}} \cdots \partial^{\alpha_{n}}
\end{aligned}
$$

where the quantity $G_{\mu \nu}^{\mathrm{CDE}}$ is

$$
G_{\mu \nu}^{\mathrm{CDE}} \equiv-i \sum_{n=0}^{\infty} \frac{n+1}{(n+2) !}\left(P_{\alpha_{1}} \cdots P_{\alpha_{n}} F_{\mu \nu}\right) \partial^{\alpha_{1}} \cdots \partial^{\alpha_{n}}
$$

with $F_{\mu \nu} \equiv-i\left[P_{\mu}, P_{\nu}\right]=g_{a} G_{\mu \nu}^{a} T^{a}$ denoting the sum over field strengths. In 
Eqs. (3.11b) and (3.12), the covariant derivatives $P_{\alpha_{1}} \cdots P_{\alpha_{n}}$ in parentheses are closed; they only act on $U_{k}$ and $F_{\mu \nu}$, respectively. Also, starting from Eq. (3.11), we reserve the shorthand notation $\partial^{\alpha}$ for a momentum derivative (as opposed to the usual position derivative):

$$
\partial^{\alpha} \equiv \frac{\partial}{\partial q_{\alpha}}
$$

With Eq. (3.11), the functional trace in Eq. (3.9) becomes

$$
-i \operatorname{Tr}\left[f\left(P_{\mu},\left\{U_{k}\right\}\right)\right]=-i \int \mathrm{d}^{d} x \int \frac{\mathrm{d}^{d} q}{(2 \pi)^{d}} \operatorname{tr}\left[f\left(P_{\mu}^{\mathrm{CDE}},\left\{U_{k}^{\mathrm{CDE}}\right\}\right)\right] .
$$

This is the central formula for the original CDE method. From this point, we can expand $f\left(P_{\mu}^{\mathrm{CDE}},\left\{U_{k}^{\mathrm{CDE}}\right\}\right)$ in powers of $U_{k}^{\mathrm{CDE}}$ and $G_{\mu \nu}^{\mathrm{CDE}}$, and then substitute in the expressions given in Eqs. (3.11b) and (3.12) to obtain the effective operators; each of them is again multiplied by a $q$-section factor. A critical feature in this expansion procedure is that all the effective operators are generated through $U_{k}^{\mathrm{CDE}}$ and $G_{\mu \nu}^{\mathrm{CDE}}$, in which all the covariant derivatives are already closed. This guarantees that no effective operators with open covariant derivatives will appear. Note that each closed covariant derivative (such as those in Eqs. (3.11b) and (3.12)) has operator dimension one and the field strength $F_{\mu \nu}$ has operator dimension two. So these expansions can be truncated according to the desired EFT operator dimension. Finally, we can carry out the loop integral $-i \int \frac{\mathrm{d}^{d} q}{(2 \pi)^{d}}$ over the $q$-sections to obtain the Wilson coefficients.

\subsection{CDE Within STrEAM}

Now let us apply the original CDE algorithm reviewed above to the supertraces targeted by STrEAM,

$$
-\left.i \mathrm{~S} \operatorname{Tr}\left[f\left(P_{\mu},\left\{U_{k}\right\}\right)\right]\right|_{\text {hard }},
$$

where $f\left(P_{\mu},\left\{U_{k}\right\}\right)$ is a product sequence of $P_{\mu}, U_{k}, \Delta_{i}$ and $\Lambda_{i}$ in the form of Eq. (2.14). Following the central formula in the original CDE, Eq. (3.14), we should make the replacements

$$
\begin{aligned}
& P_{\mu} \rightarrow P_{\mu}^{\mathrm{CDE}}=-q_{\mu}+G_{\mu \nu}^{\mathrm{CDE}} \partial^{\nu} \\
& U_{k} \rightarrow U_{k}^{\mathrm{CDE}}
\end{aligned}
$$

so that

$$
\begin{aligned}
& \Delta_{i} \quad \rightarrow \quad \Delta_{i}^{\mathrm{CDE}}=\frac{1}{\left(P_{\mu}^{\mathrm{CDE}}\right)^{2}-m_{i}^{2}}=\frac{1}{\left(-q_{\mu}+G_{\mu \nu}^{\mathrm{CDE}} \partial^{\nu}\right)^{2}-m_{i}^{2}}, \\
& \Lambda_{i} \quad \rightarrow \quad \Lambda_{i}^{\mathrm{CDE}}=\frac{1}{\not^{\mathrm{CDE}}-m_{i}}=\frac{1}{-\not p+\gamma^{\mu} G_{\mu \nu}^{\mathrm{CDE}} \partial^{\nu}-m_{i}} .
\end{aligned}
$$


Instead of using Eq. (3.17b), in STrEAM we adopt an alternative strategy to address fermionic propagators; we convert them into bosonic propagators:

$$
\begin{aligned}
\Lambda_{i} & =\frac{1}{\not P-m_{i}}=\frac{1}{\not P^{2}-m_{i}^{2}}\left(\not P+m_{i}\right)=\frac{1}{P^{2}-m_{i}^{2}-\Sigma}\left(\not P+m_{i}\right) \\
& =\left(\Delta_{i}+\Delta_{i} \Sigma \Delta_{i}+\Delta_{i} \Sigma \Delta_{i} \Sigma \Delta_{i}+\cdots\right)\left(\not P+m_{i}\right) .
\end{aligned}
$$

Here the "dipole" factor

$$
\Sigma \equiv-\frac{1}{2} \sigma^{\mu \nu} F_{\mu \nu}, \quad \text { with } \quad \sigma^{\mu \nu} \equiv \frac{i}{2}\left[\gamma^{\mu}, \gamma^{\nu}\right]
$$

can be viewed as a specific case of $U_{k}$, and it has operator dimension two. The expansion in Eq. (3.18) can be truncated according to the desired operator dimension.

After converting all the fermionic propagators $\Lambda_{i}$ to the bosonic ones $\Delta_{i}$ through Eq. (3.18), we replace $P_{\mu}, U_{k}$ (including $\Sigma$ ), and $\Delta_{i}$ with their CDE counterparts $P_{\mu}^{\mathrm{CDE}}, U_{k}^{\mathrm{CDE}}$, and $\Delta_{i}^{\mathrm{CDE}}$ as given in Eqs. (3.16) and (3.17a). We then make use of Eqs. (3.11b) and (3.12) to expand the expression into a sum of effective operators, each accompanied by a $q$-section. For the supertraces in STrEAM, the $U_{k}$ factors originate from the entries $U_{i j}[\phi], Z_{i j}^{\mu}[\phi], \bar{Z}_{i j}^{\mu}[\phi]$, etc. in the matrix $\boldsymbol{X}$ (see Eq. (2.6)), or from the dipole factor $\Sigma$ in Eq. (3.18); they have at least one power of the light fields and hence a minimum operator dimension one (but could be higher). Comparing the desired EFT operator dimension with the sum of those from $U_{k}$, we can determine where to truncate the expansion.

Finally, we perform the loop integral over the $q$-sections to obtain the Wilson coefficients. Carrying out all the $q$-derivatives and making the symmetrization of the type

$$
\begin{aligned}
q^{\mu_{1}} q^{\mu_{2}} & \rightarrow \frac{1}{d} q^{2} \eta^{\mu_{1} \mu_{2}} \\
q^{\mu_{1}} q^{\mu_{2}} q^{\mu_{3}} q^{\mu_{4}} & \rightarrow \frac{1}{d(d+2)} q^{4}\left(\eta^{\mu_{1} \mu_{2}} \eta^{\mu_{3} \mu_{4}}+\eta^{\mu_{1} \mu_{3}} \eta^{\mu_{2} \mu_{4}}+\eta^{\mu_{1} \mu_{4}} \eta^{\mu_{2} \mu_{3}}\right)
\end{aligned}
$$

one can bring the $q$-sections to (a sum of) the following form

$$
\frac{\left(q^{2}\right)^{r}}{\left(q^{2}-m_{1}^{2}\right)^{n_{1}}\left(q^{2}-m_{2}^{2}\right)^{n_{2}} \cdots\left(q^{2}-m_{k}^{2}\right)^{n_{k}}} .
$$

To obtain the hard region contributions, we need to expand this integrand into a series assuming the loop momentum $q \sim m_{\text {heavy }} \gg m_{\text {light }}$. Practically, this means identifying the light masses $m_{\text {light }}$ in Eq. (3.21), and expanding the light propagators 
as

$$
\frac{1}{q^{2}-m_{\text {light }}^{2}}=\frac{1}{q^{2}}+\frac{m_{\text {light }}^{2}}{q^{4}}+\frac{m_{\text {light }}^{4}}{q^{6}}+\cdots
$$

We truncate this expansion based on the desired total power of $m_{\text {light }}$ in the Wilson coefficients. After making this hard region expansion and truncation, the integrand can be again organized into (a sum of) the form of Eq. (3.21), but now with only heavy propagators remaining. So in the end, all the hard region loop integrals are reduced to the general form

$$
\frac{1}{16 \pi^{2}} \operatorname{LoopI}_{\left(n_{1}, \cdots, n_{k}\right)}^{(r)}\left(m_{1}^{2}, \cdots, m_{k}^{2}\right) \equiv-i \int \frac{\mathrm{d}^{d} q}{(2 \pi)^{d}} \frac{\left(q^{2}\right)^{r}}{\left(q^{2}-m_{1}^{2}\right)^{n_{1}} \cdots\left(q^{2}-m_{k}^{2}\right)^{n_{k}}}
$$

which we then evaluate with dimensional regularization and the $\overline{\mathrm{MS}}$ scheme. When there is no heavy mass propagator left in Eq. (3.23), the integral is scaleless and yields zero. When there are one or two distinct heavy masses, STrEAM provides the explicit integration results. In cases where there are three or more non-degenerate heavy masses, STrEAM leaves the loop integral in the abstract form that appears on the left hand side of Eq. (3.23).

\subsection{Implementation Summary}

In STrEAM, a functional supertrace

$$
-\left.i \mathrm{~S} \operatorname{Tr}\left[f\left(P_{\mu},\left\{U_{k}\right\}\right)\right]\right|_{\text {hard }}
$$

with $f\left(P_{\mu},\left\{U_{k}\right\}\right)$ a product sequence of $P_{\mu}, U_{k}, \Delta_{i}$ and $\Lambda_{i}$ in the form of Eq. (2.14),

$$
f=\left[\cdots\left(P_{\mu_{1}} \ldots P_{\mu_{n}}\right)\left(\Delta_{i} \text { or } \Lambda_{i}\right)\left(P_{\nu_{1}} \ldots P_{\nu_{m}}\right) U_{k} \cdots\right]
$$

is evaluated with the following steps:

- Address the "super" in STr. Assign an overall sign $+(-)$ as in Eq. (3.2) if the first propagator is $\Delta_{i}\left(\Lambda_{i}\right)$. Keep in mind that a ghost field propagator is an exception to this rule for which one needs an extra overall minus sign.

- Address fermionic propagators. Apply the relation in Eq. (3.18) to convert all the fermionic propagators $\Lambda_{i}$ into bosonic propagators $\Delta_{i}$. Truncate the expansion according to the desired operator dimension in the EFT.

- Perform original CDE. Apply the central formula of original CDE, Eq. (3.14), where $P_{\mu}, U_{k}$ (including $\Sigma$ ), and $\Delta_{i}$ are replaced with their CDE counterparts $P_{\mu}^{\mathrm{CDE}}, U_{k}^{\mathrm{CDE}}$, and $\Delta_{i}^{\mathrm{CDE}}$ given in Eqs. (3.16) and (3.17a). Then make use of Eqs. (3.11b) and (3.12) to expand the expression into a sum of effective op- 
erators, each multiplied by a $q$-section factor (a function of $q$ ). Truncate the expansion according to the desired operator dimension in the EFT.

- Perform loop integrals. For each effective operator, simplify its accompanying $q$-section into (a sum of) the form of Eq. (3.21) by carrying out the $q$ derivatives and making the symmetrization of the type in Eq. (3.20). Expand and truncate the light propagators as in Eq. (3.22). Compute the resulting integrals in the form of Eq. (3.23) to obtain the Wilson coefficients.

\subsection{A Simple Example}

In this subsection, we work out a simple example by hand as a pedagogical demonstration of the evaluation procedure in Sec. 3.3. We evaluate the supertrace

$$
T_{1} \equiv-\left.i \operatorname{STr}\left[\frac{1}{P^{2}-m_{1}^{2}} U_{1}^{[2]}\right]\right|_{\text {hard }}=-\left.i \operatorname{STr}\left[\Delta_{1} U_{1}^{[2]}\right]\right|_{\text {hard }}
$$

assuming that $m_{1}$ is a heavy mass (otherwise the hard region contribution vanishes). We evaluate this up to operator dimension six. Following the notation in Ref. [1], we have put a superscript "[2]" on $U_{1}$ to indicate that its minimum operator dimension is two.

\section{Address the "super" in STr}

In this example, the "super" part gives a positive sign because the first propagator $\Delta_{1}$ is a bosonic propagator (except when it comes from a ghost):

$$
T_{1}=-\left.i \operatorname{Tr}\left[\Delta_{1} U_{1}^{[2]}\right]\right|_{\text {hard }}
$$

\section{Address fermionic propagators}

There is no fermionic propagator $\Lambda_{i}$ to address in this example.

\section{Perform original CDE}

Following the central formula of the original CDE, Eq. (3.14), we make the replacements in Eqs. (3.16) and (3.17a) to obtain

$$
T_{1}=\left.\left[-i \int \mathrm{d}^{d} x \int \frac{\mathrm{d}^{d} q}{(2 \pi)^{d}} \operatorname{tr}\left(\Delta_{1}^{\mathrm{CDE}} U_{1}^{\mathrm{CDE}}\right)\right]\right|_{\text {hard }} .
$$

Now we need to use Eqs. (3.11b) and (3.12) to expand this expression into a sum of effective operators truncated at dimension six.

First we expand the factor $\Delta_{1}^{\mathrm{CDE}}$. Because we desire a result up to operator dimension six and $U_{1}^{\mathrm{CDE}}$ already has operator dimension two, we only need to expand 
$\Delta_{1}^{\mathrm{CDE}}$ up to operator dimension four, which is at most two powers of $G_{\mu \nu}^{\mathrm{CDE}}$ :

$$
\begin{aligned}
\Delta_{1}^{\mathrm{CDE}}= & \frac{1}{\left(-q_{\mu}+G_{\mu \nu}^{\mathrm{CDE}} \partial^{\nu}\right)^{2}-m_{1}^{2}} \\
= & \frac{1}{q^{2}-m_{1}^{2}-\left[\left(q^{\mu} G_{\mu \nu}^{\mathrm{CDE}}+G_{\mu \nu}^{\mathrm{CDE}} q^{\mu}\right) \partial^{\nu}-\eta^{\mu \nu} G_{\mu \alpha}^{\mathrm{CDE}} G_{\nu \beta}^{\mathrm{CDE}} \partial^{\alpha} \partial^{\beta}\right]} \\
= & \frac{1}{q^{2}-m_{1}^{2}}+\frac{1}{q^{2}-m_{1}^{2}}\left(q^{\mu} G_{\mu \nu}^{\mathrm{CDE}}+G_{\mu \nu}^{\mathrm{CDE}} q^{\mu}\right) \partial^{\nu} \frac{1}{q^{2}-m_{1}^{2}} \\
& +\frac{1}{q^{2}-m_{1}^{2}}\left(q^{\mu} G_{\mu \nu}^{\mathrm{CDE}}+G_{\mu \nu}^{\mathrm{CDE}} q^{\mu}\right) \partial^{\nu} \frac{1}{q^{2}-m_{1}^{2}}\left(q^{\rho} G_{\rho \sigma}^{\mathrm{CDE}}+G_{\rho \sigma}^{\mathrm{CDE}} q^{\rho}\right) \partial^{\sigma} \frac{1}{q^{2}-m_{1}^{2}} \\
& -\frac{1}{q^{2}-m_{1}^{2}} \eta^{\mu \nu} G_{\mu \alpha}^{\mathrm{CDE}} G_{\nu \beta}^{\mathrm{CDE}} \partial^{\alpha} \partial^{\beta} \frac{1}{q^{2}-m_{1}^{2}} .
\end{aligned}
$$

Now we substitute in the expression of $G_{\mu \nu}^{\mathrm{CDE}}$ in Eq. (3.12) and truncate the result at operator dimension four:

$$
\begin{aligned}
\Delta_{1}^{\mathrm{CDE}}= & \frac{1}{q^{2}-m_{1}^{2}}-i F_{\mu \nu} \frac{1}{q^{2}-m_{1}^{2}} q^{\mu} \partial^{\nu} \frac{1}{q^{2}-m_{1}^{2}}-\frac{i}{3}\left(P_{\alpha_{1}} F_{\mu \nu}\right) \frac{1}{q^{2}-m_{1}^{2}}\left(2 q^{\mu} \partial^{\alpha_{1}}+\eta^{\mu \alpha_{1}}\right) \partial^{\nu} \frac{1}{q^{2}-m_{1}^{2}} \\
& +\frac{1}{4} F_{\mu \nu} F_{\rho \sigma}\left(\eta^{\mu \rho} \frac{1}{q^{2}-m_{1}^{2}} \partial^{\nu} \partial^{\sigma} \frac{1}{q^{2}-m_{1}^{2}}-4 \frac{1}{q^{2}-m_{1}^{2}} q^{\mu} \partial^{\nu} \frac{1}{q^{2}-m_{1}^{2}} q^{\rho} \partial^{\sigma} \frac{1}{q^{2}-m_{1}^{2}}\right) \\
& -\frac{i}{4}\left(P_{\alpha_{1}} P_{\alpha_{2}} F_{\mu \nu}\right) \frac{1}{q^{2}-m_{1}^{2}}\left(q^{\mu} \partial^{\alpha_{2}}+\eta^{\mu \alpha_{2}}\right) \partial^{\nu} \partial^{\alpha_{1}} \frac{1}{q^{2}-m_{1}^{2}}
\end{aligned}
$$

Next, we expand the factor $U_{1}^{\mathrm{CDE}}$ using Eq. (3.11b). Note that $U_{1}^{\mathrm{CDE}}$ in this example is the last factor in the expression, so all of its momentum derivatives $\partial^{\alpha_{i}}$ will be acting on the unity function $\mathbb{1}$ to yield zero; only the first term in Eq. (3.11b) survives, and we can set

$$
U_{1}^{\mathrm{CDE}}=U_{1}
$$

Substituting Eqs. (3.30) and (3.31) into Eq. (3.28), we obtain

$$
\begin{aligned}
T_{1}=-i \int & \mathrm{d}^{d} x \int \frac{\mathrm{d}^{d} q}{(2 \pi)^{d}} \operatorname{tr}\left\{U_{1}\left[\frac{1}{q^{2}-m_{1}^{2}}\right]+F_{\mu \nu} U_{1}\left[-i \frac{1}{q^{2}-m_{1}^{2}} q^{\mu} \partial^{\nu} \frac{1}{q^{2}-m_{1}^{2}}\right]\right. \\
& +\left(P_{\alpha_{1}} F_{\mu \nu}\right) U_{1}\left[-\frac{i}{3} \frac{1}{q^{2}-m_{1}^{2}}\left(2 q^{\mu} \partial^{\alpha_{1}}+\eta^{\mu \alpha_{1}}\right) \partial^{\nu} \frac{1}{q^{2}-m_{1}^{2}}\right] \\
& +F_{\mu \nu} F_{\rho \sigma} U_{1}\left[\frac{1}{4} \eta^{\mu \rho} \frac{1}{q^{2}-m_{1}^{2}} \partial^{\nu} \partial^{\sigma} \frac{1}{q^{2}-m_{1}^{2}}-\frac{1}{q^{2}-m_{1}^{2}} q^{\mu} \partial^{\nu} \frac{1}{q^{2}-m_{1}^{2}} q^{\rho} \partial^{\sigma} \frac{1}{q^{2}-m_{1}^{2}}\right] \\
& \left.+\left(P_{\alpha_{1}} P_{\alpha_{2}} F_{\mu \nu}\right) U_{1}\left[-\frac{i}{4} \frac{1}{q^{2}-m_{1}^{2}}\left(q^{\mu} \partial^{\alpha_{2}}+\eta^{\mu \alpha_{2}}\right) \partial^{\nu} \partial^{\alpha_{1}} \frac{1}{q^{2}-m_{1}^{2}}\right]\right\}\left.\right|_{\text {hard }}
\end{aligned}
$$


We see that up to operator dimension six, there are five effective operators:

$$
\operatorname{tr}\left(U_{1}\right), \operatorname{tr}\left(F_{\mu \nu} U_{1}\right), \operatorname{tr}\left[\left(P_{\alpha_{1}} F_{\mu \nu}\right) U_{1}\right], \operatorname{tr}\left(F_{\mu \nu} F_{\rho \sigma} U_{1}\right), \operatorname{tr}\left[\left(P_{\alpha_{1}} P_{\alpha_{2}} F_{\mu \nu}\right) U_{1}\right]
$$

each multiplied by a $q$-section factor gathered in the square brackets in Eq. (3.32).

\section{Perform loop integrals}

We carry out the $q$-derivatives in the $q$-sections in Eq. (3.32) and compute the hard region contributions to the loop integrals to obtain the Wilson coefficients. Only two of the five integrals are nonzero; the end result is

$$
T_{1}=\int \mathrm{d}^{d} x \frac{1}{16 \pi^{2}} \operatorname{tr}\left[m_{1}^{2}\left(1-\log \frac{m_{1}^{2}}{\mu^{2}}\right) U_{1}+\frac{1}{12 m_{1}^{2}} F_{\mu \nu} F^{\mu \nu} U_{1}\right],
$$

reproducing Eq. (C.1) in Ref. [1].

This completes the demonstration of the evaluation procedure detailed in Sec. 3.3. Clearly, the same procedure applies to any functional supertrace targeted by STrEAM, with $f\left(P_{\mu},\left\{U_{k}\right\}\right)$ in the form of Eq. (2.14). For more complicated expressions of $f$, the CDE steps shown in Eqs. (3.29)-(3.31) are more involved, and the resulting expression in Eq. (3.32) would contain more effective operators and more complicated $q$-sections. Accordingly, the $q$-derivatives and the loop integral $-i \int \frac{\mathrm{d}^{d} q}{(2 \pi)^{d}}$ are also more tedious to carry out. Nevertheless, these steps are completely algorithmic. STrEAM automates them and provides results analogous to Eq. (3.34) as its output. For example, all the other supertraces listed in App. C in Ref. [1] are also readily reproduced with STrEAM. In practical EFT matching calculations, one then substitutes in the explicit expressions for $\left\{U_{k}\right\}$ and evaluates the remaining trace ("tr" in Eq. (3.34)) as explained in Ref. [1]. One can further translate the result into an EFT operator basis via integration by parts, equations of motion and operator identities if desired.

\section{$4 \quad$ STrEAM Manual}

In this section, we provide a user manual for STrEAM. We will go over some basics of using this package and show a few concrete examples.

\section{Downloading and loading}

STrEAM is a Mathematica package publicly available on GitHub ( ) [34]. Once the file "STrEAM.m" is placed in the user's directory of choice /path/to/package/, it can be loaded in Mathematica with the usual command:

$$
\ln [1]:=<<" / \text { path/to/package/STrEAM.m"; }
$$




\section{Main functions}

STrEAM is a compact package with essentially a single main function SuperTrace that carries out the procedure summarized in Sec. 3.3. Once the package is loaded, the user can readily execute this main function:

$$
\ln [2]:=\text { SuperTrace [dim, flist] }
$$

As indicated above, it has two mandatory arguments. dim is an Integer that specifies the desired operator dimension in the evaluation result. $f l$ ist is a List that specifies the functional operator $f\left(P_{\mu},\left\{U_{k}\right\}\right)$ to be traced over; it consists of $\mathrm{P}_{\mu}, \mathrm{U}_{\mathrm{k}}, \Delta_{\mathrm{i}}$, and $\Lambda_{i}$, organized in the form of Eq. (2.14). This is the main input to the function, and a few remarks are in order:

- The symbols P, $\Delta$, and $\Lambda$ are reserved in STrEAM, such that the package can recognize these key elements in $f$ list. On the other hand, the symbol $U$ is not reserved, and the user can choose their own symbols for the elements $U_{k}$.

- In flist, each $\mathrm{P}_{\mu}$ must come with a subscript as its Lorentz index. One can choose their own symbol for it (but the same Lorentz index should not appear more than twice). The Lorentz indices $\mu_{\mathrm{i}}$ are reserved in STrEAM as dummy indices generated in the expansions as well as in the final outputs. If they were encountered in the input, they would be automatically replaced with $\nu_{j}$ with some proper integer $j$.

- Recall from Eq. (2.12) that each propagator $\Delta_{i}$ or $\Lambda_{i}$ must come with a subscript $i$, indicating its mass $m_{i}$. These mass labels are non-negative integers, and will be used for specifying the list of heavy masses. This is crucial because STrEAM evaluates the hard region contributions to supertraces. The label " 0 " is reserved for massless propagators, i.e., we have stipulated $m_{0}=0$ ( $c f$. Eq. (2.12)):

$$
\Delta_{0} \equiv \frac{1}{P^{2}}, \quad \Lambda_{0} \equiv \frac{1}{\not P}
$$

- As explained in Sec. 2, in order to accommodate the log-type supertraces via Eq. (2.15), we allow the last $U$ factor in $f\left(P_{\mu},\left\{U_{k}\right\}\right)$ to be trivial. This is implemented by allowing the very last $U_{k}$ factor in $f l i s t$ to be absent. 
There are also a few options for SuperTrace:

\begin{tabular}{|ccl|}
\hline Option & Default & Description \\
\hline Udimlist & $\{1, \ldots, 1\}$ & Minimum operator dimensions of $\left\{U_{k}\right\}$ \\
Heavylist & $\{1\}$ & Heavy mass labels \\
SoftOrd & 0 & Additional power(s) of $m_{\text {light }} / m_{\text {heavy }}$ \\
No $\gamma$ inU & False & No Dirac matrices $\gamma_{\mu}$ in $\left\{U_{k}\right\}$ \\
display & False & Print result \\
\hline
\end{tabular}

As explained in Sec. 3.2, the minimum operator dimensions of the $U_{\mathrm{k}}$ factors, gathered in Udimlist, are needed to determine when to truncate the CDE. The default setting is that they are all unity. Heavylist specifies the list of heavy masses through their (positive integer) labels; this is crucial for identifying the hard region contributions. The default setting is that only $m_{1}$ is heavy. Softord is a non-negative integer. When it is set to the default value 0 , the Wilson coefficient of an effective operator with operator dimension $\operatorname{dim}_{\mathcal{O}}$ will be computed up to $\left(\operatorname{dim}-\operatorname{dim}_{\mathcal{O}}\right)$ powers of $m_{\text {light }} / m_{\text {heavy }}$. If additional powers are desired, one can specify a positive SoftOrd. No $\gamma$ inU can be used to simplify the result when there are no Dirac matrices $\gamma_{\mu}$ in the $\mathrm{U}_{\mathrm{k}}$ factors. The option display controls whether to print the result.

Let us consider a very simple example - the supertrace discussed in Sec. 3.4:

$$
T_{1} \equiv-\left.i \operatorname{STr}\left[\Delta_{1} U_{1}^{[2]}\right]\right|_{\text {hard }}
$$

To evaluate this supertrace up to operator dimension six, one simply runs

$$
\ln [2]:=\text { SuperTrace }\left[6,\left\{\Delta_{1}, U_{1}\right\}, \operatorname{Udimlist} \rightarrow\{2\}\right]
$$

SuperTrace returns the evaluation result as a list of terms, with each term in the following form:

$$
\{\text { coeff, oper, dim }\}
$$

where coeff and oper are lists themselves that contain the Wilson coefficient (multiplied by $16 \pi^{2}$ ) and the effective operator respectively; dim records the operator dimension of the term. For instance, the above example yields an output with two such terms: 


$$
\begin{aligned}
\text { Out [2] }=\{ & \left\{\left\{\left\{\mathrm{m}_{1}^{2}\left(1-\log \left[\frac{\mathrm{m}_{1}^{2}}{\mu^{2}}\right]\right)\right\}\right\},\left\{\left\{\mathrm{U}_{1}\right\}\right\}, 2\right\}, \\
& \left.\left\{\left\{\left\{\frac{1}{12 \mathrm{~m}_{1}^{2}}\right\}\right\},\left\{\left\{\mathrm{F}_{\mu_{1}, \mu_{2}}\right\},\left\{\mathrm{F}_{\mu_{1}, \mu_{2}}\right\},\left\{\mathrm{U}_{1}\right\}\right\}, 6\right\}\right\}
\end{aligned}
$$

When the option display $\rightarrow$ True is used, SuperTrace will print the evaluation result in TableForm, together with the input supertrace. Again for the example in Eq. (4.2):

$$
\ln [3]:=\text { SuperTrace }\left[6,\left\{\Delta_{1}, U_{1}\right\}, \text { Udimlist } \rightarrow\{2\} \text {, display } \rightarrow \text { True }\right] \text {; }
$$

will print

$$
\begin{array}{lll}
-\left.i \operatorname{STr}\left[\frac{1}{\mathrm{P}^{2}-\mathrm{m}_{1}^{2}} \mathrm{U}_{1}\right]\right|_{\text {hard }}=\int \mathrm{d}^{4} \mathrm{x} \frac{1}{16 \pi^{2}} \operatorname{tr}\{ & (\mathrm{dim}-2) \\
\mathrm{m}_{1}^{2}\left(1-\log \left[\frac{\mathrm{m}_{1}^{2}}{\mu^{2}}\right]\right) & \left(\mathrm{U}_{1}\right) \\
\frac{1}{12 \mathrm{~m}_{1}^{2}} & \left(\mathrm{~F}_{\mu_{1} \mu_{2}}\right)\left(\mathrm{F}_{\mu_{1} \mu_{2}}\right)\left(\mathrm{U}_{1}\right) \\
\} &
\end{array}
$$

We recommend using this option for checking the result in a more readable format.

There is also an alternative function SuperTraceFromExpr, which is a slight variant of SuperTrace that takes an expression fexpr, instead of a List flist, as the input for specifying the functional operator $f\left(P_{\mu},\left\{U_{k}\right\}\right)$ :

$$
\ln [4]:=\text { SuperTraceFromExpr [dim, fexpr }]
$$

It has the same set of options as SuperTrace. The input expression fexpr is obtained by putting together the elements in flist with NonCommutativeMultiply $(* *)$. For instance, to evaluate the same example in Eq. (4.2), one can execute

$$
\operatorname{In}[4]:=\text { SuperTraceFromExpr }\left[6, \Delta_{1} * * U_{1}, \text { Udimlist } \rightarrow\{2\}, \text { display } \rightarrow \text { True }\right] \text {; }
$$

The output of SuperTraceFromExpr is the same as that of SuperTrace.

\section{Reserved variables}

As mentioned before, in STrEAM the symbols $\mathrm{P}, \Delta$, and $\Lambda$ are reserved for input recognition. The Lorentz indices $\mu_{\mathrm{i}}$ are reserved for calculation purposes. $\mathrm{P}$ and $\mu_{\mathrm{i}}$ are also used in the outputs. Apart from these, the following symbols are also reserved for special meanings: 


\begin{tabular}{|cl|}
\hline Reserved symbol & Meaning \\
\hline $\mathrm{m}$ & Particle masses $m_{i}$ \\
$\mathrm{~d}$ & Spacetime dimension in dimensional regularization \\
$\eta$ & Spacetime metric $\eta_{\mu \nu}=\operatorname{diag}(1,-1,-1,-1)$ \\
$\varepsilon$ & Levi-Civita symbol $\varepsilon_{\mu \nu \rho \sigma}$ with $\varepsilon_{0123}=-1$ \\
$\mathrm{~F}$ & Field strength $F_{\mu \nu} \equiv-i\left[P_{\mu}, P_{\nu}\right]=g_{a} G_{\mu \nu}^{a} T^{a}$ \\
$\gamma$ & Dirac matrices $\gamma_{\mu}$ \\
$\sigma \mathrm{F}$ & $\sigma^{\mu \nu} F_{\mu \nu}$ in the dipole factor (see Eq. $\left.(3.19)\right)$ \\
Pslash & $\not P=\gamma^{\mu} P_{\mu}$ \\
LoopI & Loop integral in Eq. (3.23) for $\geq 3$ heavy masses \\
\hline
\end{tabular}

\section{Additional examples}

Finally, we show a few selected input examples to better illustrate the syntax. We will not include their outputs or prints here. These results, as well as more demonstration examples, are collected in a Mathematica notebook "STrEAM_examples.nb" ○ [34]. For each example below, one can add the option display $\rightarrow$ True if desired.

- Supertraces converted from log-type via Eq. (2.15),

$$
-\left.i \mathrm{~S} \operatorname{Tr}\left[\frac{1}{P^{2}-m_{1}^{2}}\right]\right|_{\text {hard }}, \quad-\left.i \mathrm{~S} \operatorname{Tr}\left[\frac{1}{\not P-m_{1}}\right]\right|_{\text {hard }}
$$

can be evaluated up to operator dimension six with

$$
\begin{aligned}
& \operatorname{In}[5]:=\operatorname{SuperTrace}\left[6,\left\{\Delta_{1}\right\}\right] \\
& \operatorname{In}[6]:=\text { SuperTrace }\left[6,\left\{\Lambda_{1}\right\}, \text { No } \gamma \text { inU } \rightarrow \text { True }\right]
\end{aligned}
$$

Note that we have turned on the option No $\gamma$ inU $\rightarrow$ True for the fermionic one.

- A supertrace with both heavy and light propagators $\left(m_{1}\right.$ and $\left.m_{2}\right)$, such as

$$
-\left.i \mathrm{~S} \operatorname{Tr}\left[\frac{1}{P^{2}-m_{1}^{2}} U_{1}^{[1]} \frac{1}{P^{2}-m_{2}^{2}} U_{2}^{[1]} \frac{1}{P^{2}-m_{1}^{2}} U_{3}^{[1]} \frac{1}{P^{2}-m_{2}^{2}} U_{4}^{[1]}\right]\right|_{\text {hard }}
$$

can be evaluated up to operator dimension six with 


$$
\operatorname{In}[7]:=\operatorname{SuperTrace}\left[6,\left\{\Delta_{1}, \mathrm{U}_{1}, \Delta_{2}, \mathrm{U}_{2}, \Delta_{1}, \mathrm{U}_{3}, \Delta_{2}, \mathrm{U}_{4}\right\}\right]
$$

or equivalently with

$$
\ln [7]:=\text { SuperTraceFromExpr }\left[6, \Delta_{1} * * \mathrm{U}_{1} * * \Delta_{2} * * \mathrm{U}_{2} * * \Delta_{1} * * \mathrm{U}_{3} * * \Delta_{2} * * \mathrm{U}_{4}\right]
$$

- A supertrace with explicit open covariant derivatives, such as

$$
-\left.i \operatorname{STr}\left[\frac{1}{P^{2}-m_{1}^{2}} U_{1}^{[1]} \frac{1}{P^{2}-m_{2}^{2}} P_{\mu} Z^{\mu[1]} \frac{1}{P^{2}} U_{3}^{[2]} \frac{1}{P^{2}-m_{2}^{2}} U_{4}^{[1]}\right]\right|_{\text {hard }},
$$

can be evaluated up to operator dimension six with

$$
\begin{aligned}
& \operatorname{In}[8]:=\text { SuperTrace }\left[6,\left\{\Delta_{1}, \mathrm{U}_{1}, \Delta_{2}, \mathrm{P}_{\nu}, \mathrm{Z}_{\nu}, \Delta_{0}, \mathrm{U}_{3}, \Delta_{2}, \mathrm{U}_{4}\right\}\right. \text {, } \\
& \text { Udimlist } \rightarrow\{1,1,2,1\}]
\end{aligned}
$$

- A supertrace with fermionic propagators, such as

$$
-\left.i \operatorname{STr}\left[\frac{1}{P^{2}-m_{1}^{2}} U_{1}^{[1]} \frac{1}{P^{2}-m_{2}^{2}} U_{2}^{[3 / 2]} \frac{1}{\not P} U_{3}^{[3 / 2]} \frac{1}{P^{2}-m_{2}^{2}} U_{4}^{[1]}\right]\right|_{\text {hard }}
$$

can be evaluated up to operator dimension six with

$$
\begin{gathered}
\operatorname{In}[9]:=\text { SuperTrace }\left[6,\left\{\Delta_{1}, \mathrm{U}_{1}, \Delta_{2}, \mathrm{U}_{2}, \Lambda_{0}, \mathrm{U}_{3}, \Delta_{2}, \mathrm{U}_{4}\right\},\right. \\
\text { Udimlist } \left.\rightarrow\left\{1, \frac{3}{2}, \frac{3}{2}, 1\right\}\right]
\end{gathered}
$$

\section{Acknowledgments}

We thank Javier Fuentes-Martín, Matthias König, Julie Pagès, Anders Eller Thomsen, and Felix Wilsch for communications about their related work [50] and crosschecks. T.C. and X.L. are supported by the U.S. Department of Energy, under grant number DE-SC0011640. Z.Z.'s work was supported in part by the U.S. Department of Energy, Office of Science, Office of High Energy Physics, under Award Number DE-AC02-05CH11231.

\section{References}

[1] T. Cohen, X. Lu, and Z. Zhang, "Functional Prescription for EFT Matching," arXiv:2011.02484 [hep-ph].

[2] I. Z. Rothstein, "TASI lectures on effective field theories," 8, 2003. arXiv:hep-ph/0308266.

[3] W. Skiba, "Effective Field Theory and Precision Electroweak Measurements," in Theoretical Advanced Study Institute in Elementary Particle Physics: Physics of the Large and the Small, pp. 5-70. 2011. arXiv:1006.2142 [hep-ph]. 
[4] A. A. Petrov and A. E. Blechman, Effective Field Theories. WSP, 2016.

[5] LHC Higgs Cross Section Working Group Collaboration, D. de Florian et al., "Handbook of LHC Higgs Cross Sections: 4. Deciphering the Nature of the Higgs Sector," arXiv:1610.07922 [hep-ph].

[6] I. Brivio and M. Trott, "The Standard Model as an Effective Field Theory," Phys. Rept. 793 (2019) 1-98, arXiv:1706.08945 [hep-ph].

[7] A. V. Manohar, "Introduction to Effective Field Theories," Les Houches Lect. Notes 108 (2020), arXiv: 1804.05863 [hep-ph].

[8] M. Neubert, "Les Houches Lectures on Renormalization Theory and Effective Field Theories," Les Houches Lect. Notes 108 (2020), arXiv:1901.06573 [hep-ph].

[9] T. Cohen, "As Scales Become Separated: Lectures on Effective Field Theory," PoS TASI2018 (2019) 011, arXiv: 1903.03622 [hep-ph].

[10] R. Penco, "An Introduction to Effective Field Theories," arXiv:2006.16285 [hep-th].

[11] M. Gaillard, "The Effective One Loop Lagrangian With Derivative Couplings," Nucl. Phys. B 268 (1986) 669-692.

[12] L.-H. Chan, "Derivative Expansion for the One Loop Effective Actions With Internal Symmetry," Phys. Rev. Lett. 57 (1986) 1199.

[13] O. Cheyette, "Effective Action for the Standard Model With Large Higgs Mass," Nucl. Phys. B 297 (1988) 183-204.

[14] B. Henning, X. Lu, and H. Murayama, "What do precision Higgs measurements buy us?," arXiv:1404.1058 [hep-ph].

[15] B. Henning, X. Lu, and H. Murayama, "How to use the Standard Model effective field theory," JHEP 01 (2016) 023, arXiv:1412.1837 [hep-ph].

[16] A. Drozd, J. Ellis, J. Quevillon, and T. You, "The Universal One-Loop Effective Action," JHEP 03 (2016) 180, arXiv:1512.03003 [hep-ph].

[17] C.-W. Chiang and R. Huo, "Standard Model Effective Field Theory: Integrating out a Generic Scalar," JHEP 09 (2015) 152, arXiv:1505.06334 [hep-ph].

[18] R. Huo, "Standard Model Effective Field Theory: Integrating out Vector-Like Fermions," JHEP 09 (2015) 037, arXiv: 1506.00840 [hep-ph].

[19] R. Huo, "Effective Field Theory of Integrating out Sfermions in the MSSM: Complete One-Loop Analysis," Phys. Rev. D 97 (2018) no. 7, 075013, arXiv:1509.05942 [hep-ph].

[20] B. Henning, X. Lu, and H. Murayama, "One-loop Matching and Running with Covariant Derivative Expansion," JHEP 01 (2018) 123, arXiv:1604.01019 [hep-ph].

[21] S. A. R. Ellis, J. Quevillon, T. You, and Z. Zhang, "Mixed heavy-light matching in 
the Universal One-Loop Effective Action," Phys. Lett. B 762 (2016) 166-176, arXiv: 1604.02445 [hep-ph].

[22] J. Fuentes-Martin, J. Portoles, and P. Ruiz-Femenia, "Integrating out heavy particles with functional methods: a simplified framework," JHEP 09 (2016) 156, arXiv:1607.02142 [hep-ph].

[23] Z. Zhang, "Covariant diagrams for one-loop matching," JHEP 05 (2017) 152, arXiv:1610.00710 [hep-ph].

[24] S. A. R. Ellis, J. Quevillon, T. You, and Z. Zhang, "Extending the Universal One-Loop Effective Action: Heavy-Light Coefficients," JHEP 08 (2017) 054, arXiv:1706.07765 [hep-ph].

[25] J. D. Wells and Z. Zhang, "Effective field theory approach to trans-TeV supersymmetry: covariant matching, Yukawa unification and Higgs couplings," JHEP 05 (2018) 182, arXiv:1711.04774 [hep-ph].

[26] H. Han, R. Huo, M. Jiang, and J. Shu, "Standard Model Effective Field Theory: Integrating out Neutralinos and Charginos in the MSSM," Phys. Rev. D 97 (2018) no. 9, 095003, arXiv:1712.07825 [hep-ph].

[27] B. Summ and A. Voigt, "Extending the Universal One-Loop Effective Action by Regularization Scheme Translating Operators," JHEP 08 (2018) 026, arXiv:1806.05171 [hep-ph].

[28] M. Jiang, N. Craig, Y.-Y. Li, and D. Sutherland, "Complete One-Loop Matching for a Singlet Scalar in the Standard Model EFT," JHEP 02 (2019) 031, arXiv: 1811.08878 [hep-ph].

[29] M. Krämer, B. Summ, and A. Voigt, "Completing the scalar and fermionic Universal One-Loop Effective Action," JHEP 01 (2020) 079, arXiv:1908.04798 [hep-ph].

[30] T. Cohen, M. Freytsis, and X. Lu, "Functional Methods for Heavy Quark Effective Theory," JHEP 06 (2020) 164, arXiv:1912.08814 [hep-ph].

[31] S. A. Ellis, J. Quevillon, P. N. H. Vuong, T. You, and Z. Zhang, "The Fermionic Universal One-Loop Effective Action," arXiv:2006.16260 [hep-ph].

[32] A. Angelescu and P. Huang, "Integrating Out New Fermions at One Loop," arXiv:2006.16532 [hep-ph].

[33] T. Cohen, N. Craig, X. Lu, and D. Sutherland, "Is SMEFT Enough?," arXiv:2008.08597 [hep-ph].

[34] https://www.github.com/EFTMatching/STrEAM $($.

[35] A. Celis, J. Fuentes-Martin, A. Vicente, and J. Virto, "DsixTools: The Standard Model Effective Field Theory Toolkit," Eur. Phys. J. C 77 (2017) no. 6, 405, arXiv: 1704.04504 [hep-ph].

[36] I. Brivio, Y. Jiang, and M. Trott, "The SMEFTsim package, theory and tools," JHEP 12 (2017) 070, arXiv:1709.06492 [hep-ph]. 
[37] J. C. Criado, "MatchingTools: a Python library for symbolic effective field theory calculations," Comput. Phys. Commun. 227 (2018) 42-50, arXiv:1710.06445 [hep-ph] .

[38] J. Aebischer, J. Kumar, and D. M. Straub, "Wilson: a Python package for the running and matching of Wilson coefficients above and below the electroweak scale," Eur. Phys. J. C 78 (2018) no. 12, 1026, arXiv:1804.05033 [hep-ph].

[39] B. Gripaios and D. Sutherland, "DEFT: A program for operators in EFT," JHEP 01 (2019) 128, arXiv:1807.07546 [hep-ph].

[40] S. Das Bakshi, J. Chakrabortty, and S. K. Patra, "CoDEx: Wilson coefficient calculator connecting SMEFT to UV theory," Eur. Phys. J. C 79 (2019) no. 1, 21, arXiv:1808.04403 [hep-ph].

[41] J. Aebischer, J. Kumar, P. Stangl, and D. M. Straub, "A Global Likelihood for Precision Constraints and Flavour Anomalies," Eur. Phys. J. C 79 (2019) no. 6, 509, arXiv:1810.07698 [hep-ph].

[42] D. M. Straub, "flavio: a Python package for flavour and precision phenomenology in the Standard Model and beyond," arXiv:1810.08132 [hep-ph].

[43] J. C. Criado, "BasisGen: automatic generation of operator bases," Eur. Phys. J. C 79 (2019) no. 3, 256, arXiv:1901.03501 [hep-ph].

[44] N. P. Hartland, F. Maltoni, E. R. Nocera, J. Rojo, E. Slade, E. Vryonidou, and C. Zhang, "A Monte Carlo global analysis of the Standard Model Effective Field Theory: the top quark sector," JHEP 04 (2019) 100, arXiv:1901.05965 [hep-ph].

[45] A. Dedes, M. Paraskevas, J. Rosiek, K. Suxho, and L. Trifyllis, "SmeftFR - Feynman rules generator for the Standard Model Effective Field Theory," Comput. Phys. Commun. 247 (2020) 106931, arXiv:1904.03204 [hep-ph].

[46] J. Fuentes-Martin, P. Ruiz-Femenia, A. Vicente, and J. Virto, "DsixTools 2.0: The Effective Field Theory Toolkit," arXiv:2010.16341 [hep-ph].

[47] D. van Dyk et al., "EOS - A HEP program for Flavor Observables." https://eos.github.io, 2016.

[48] C. Anastasiou, A. Carmona, A. Lazopoulos, and J. Santiago, "MatchMaker." https://indico.cern.ch/event/787665/contributions/3374418.

[49] J. Aebischer, M. Fael, A. Lenz, M. Spannowsky, and J. Virto, eds., Computing Tools for the SMEFT. 10, 2019. arXiv:1910.11003 [hep-ph].

[50] J. Fuentes-Martín, M. König, J. Pagès, A. E. Thomsen, and F. Wilsch, "SuperTracer: A calculator of functional supertraces for one-loop EFT matching," 2020.

[51] M. Beneke and V. A. Smirnov, "Asymptotic expansion of Feynman integrals near threshold," Nucl. Phys. B522 (1998) 321-344, arXiv:hep-ph/9711391 [hep-ph].

[52] V. A. Smirnov, "Applied asymptotic expansions in momenta and masses," Springer Tracts Mod. Phys. 177 (2002) 1-262. 